\title{
Morphometric evaluation of nitric oxide synthase isoforms and their cytokine regulators predict pulmonary dysfunction and survival in systemic sclerosis
}

\author{
E.R. Parra, A.C. Aguiar Junior, L.O. Silva, H.S.P. Souza, J.D. Espinoza and V.L. Capelozzi \\ Departamento de Patologia, Faculdade de Medicina, Universidade de São Paulo, São Paulo, SP, Brasil
}

\begin{abstract}
Because histopathological changes in the lungs of patients with systemic sclerosis (SSc) are consistent with alveolar and vessel cell damage, we presume that this interaction can be characterized by analyzing the expression of proteins regulating nitric oxide (NO) and plasminogen activator inhibitor-1 (PAl-1) synthesis. To validate the importance of alveolar-vascular interactions and to explore the quantitative relationship between these factors and other clinical data, we studied these markers in 23 cases of SSc nonspecific interstitial pneumonia (SSc-NSIP). We used immunohistochemistry and morphometry to evaluate the amount of cells in alveolar septa and vessels staining for NO synthase (NOS) and PAI-1, and the outcomes of our study were cellular and fibrotic NSIP, pulmonary function tests, and survival time until death. General linear model analysis demonstrated that staining for septal inducible NOS (iNOS) related significantly to staining of septal cells for interleukin (IL)-4 and to septal IL-13. In univariate analysis, higher levels of septal and vascular cells staining for iNOS were associated with a smaller percentage of septal and vascular cells expressing fibroblast growth factor and myofibroblast proliferation, respectively. Multivariate Cox model analysis demonstrated that, after controlling for SSc-NSIP histological patterns, just three variables were significantly associated with survival time: septal iNOS $(P=0.04)$, septal IL-13 $(P=0.03)$, and septal basic fibroblast growth factor (bFGF; $P=0.02)$. Augmented NOS, IL13, and bFGF in SSc-NSIP histological patterns suggest a possible functional role for iNOS in SSc. In addition, the extent of iNOS, PAI-1, and IL-4 staining in alveolar septa and vessels provides a possible independent diagnostic measure for the degree of pulmonary dysfunction and fibrosis with an impact on the survival of patients with SSc.
\end{abstract}

Key words: Systemic sclerosis; Nitric oxide synthase isoforms; Cytokines; Morphometry; Pulmonary function tests and survival

\section{Introduction}

Systemic sclerosis (SSc) is an autoimmune disease characterized by vascular abnormalities, fibrosis of the skin, musculoskeletal manifestations, and internal organ involvement (1). Pulmonary involvement in SSc in the form of cellular or fibrotic nonspecific interstitial pneumonia (NSIP) occurs in $25-90 \%$ of patients, depending on the sensitivity of the evaluation (2-6), and is a significant cause of morbidity and mortality in this patient population (2-7). Consequently, there is great interest to identify which NSIP groups are likely to progress to a more fibrotic pattern that may result in shorter patient survival. In addition, identification of these specific NSIP groups after surgical lung biopsy may allow for optimal treatment approaches. In this regard many have studied biological markers in alveolar as well as in vascular compartments to discover what might relate with the progression of fibrosis or treatment responses, or to tumor recurrence and shortened survival (8-12). Because sclerodermaassociated fibrotic lung disease is the phenotypic consequence of the interactions between epithelial and mesenchymal components (such as endothelial cells and myofibroblasts), currently much interest is focused on the influence of proliferative factors on growth, activation, and replication of these components. SSc is thought to be a consequence of the aberrant regulation of endothelial tissue, resulting in both vascular damage and subsequent tissue damage. Thus, several interleukins (IL4, IL-6, IL-8, and IL-13) and growth factors [transforming growth factor beta (TGF- $\beta$ ), platelet-derived growth factor (PDGF), tumor necrosis factor alpha (TNF- $\alpha)$, insulin-like growth factors (IGFs), basic fibroblast growth factor (bFGF), and interferon gamma (IFN- $\gamma$ )] released from

Correspondence: E.R. Parra, Departamento de Patologia, Faculdade de Medicina, USP, Av. Dr. Arnaldo, 455, Sala 1143, $01246-903$ São Paulo, SP, Brasil. Fax: +55-11-3064-2744. E-mail: erparra20003@yahoo.com.br 
inflammatory cells, endothelial cells, fibroblasts, and other cells in the lung have been implicated in the initiation and maintenance of the fibrotic process $(13,14)$. In addition, a group of nitric oxide (NO) synthase (NOS) isoforms has been targeted as potentially useful vascular markers of dysfunction $(15,16)$. Among these, constitutively expressed endothelial NO synthase (eNOS) and plasminogen activator inhibitor-1 (PAl-1) have shown promise. In many pathological states, most notably reperfusion injuries, dysregulation of inducible NOS (iNOS) and PAI-1 result in endothelial damage, thus leading to excessive levels of NO. Excessive levels of NO react with superoxides to form peroxynitrite and highly reactive hydroxyl radicals, which in turn result in cell injury and apoptosis (17). As the histopathological changes in the lungs of patients with SSc are consistent with alveolar and vessel cell damage (18-20), we presume that this interaction can be characterized by analyzing the expression of proteins regulating NO synthesis. To validate the importance of alveolar-vascular interactions and to explore the quantitative relationship between these factors and the outcome, as well as the relationship between these factors and other clinical data and pulmonary function tests, we studied these markers in 23 SSc-NSIP cases.

\section{Patients and Methods}

Between January 2002 and July 2004, 23 consecutive patients with SSc and interstitial lung disease (ILD) shown by high-resolution computed tomography (HRCT) were submitted to an open lung biopsy at the Hospital das Clínicas, Universidade de São Paulo (21). All patients were women (mean age $\pm S D, 44.89 \pm 8.74$ years) who fulfilled the diagnostic and subtype criteria for SSc $(22,23)$. Open lung biopsy was performed by formal thoracotomy avoiding honeycombing areas. All 23 patients signed a free informed consent (No. 0960/08) form, and the study was approved by the Hospital Ethics and Scientific Committees.

Analysis of the clinical records was performed for all patients. The disease duration was established from the first symptom of the disease except for Raynaud's phenomenon. Skin thickness was assessed using the modified Rodnan Skin Score (MRSS) (24), consisting of clinical palpation in 17 body areas on a 0-3 basis and the sum of the scores in all 17 areas. HRCT and pulmonary function tests were performed within a period of up to 3 months before the biopsy. Disease duration (from the onset of Raynaud's phenomenon) and MRSS to score cutaneous fibrosis were analyzed. All eligible patients were submitted to blood tests immediately before the start of the study (complete blood count, urinalysis, liver enzymes, renal function tests, and anti-topoisomerase antibody). They were followed monthly before cyclophosphamide infusion with regular blood tests, and the dosage was adjusted if the total leukocyte count fell below $3000 / \mathrm{mm}^{3}$.
Lung function tests [diffusing capacity of the lung for carbon monoxide corrected for hemoglobin concentration (DLCO$\mathrm{Hb}$ ), forced vital capacity (FVC), forced expiratory volume in the first second (FEV1), and total lung capacity (TLC)] were performed before (up to 3 months), after 12 months of treatment, and after 3 years from the end of the study. MRSS was scored before treatment, on months 6 and 12 of treatment, and also after 3 years from the end of the study. The primary end point was to evaluate changes in NOS and PAI-1 and to analyze differences between the two groups: cellular SSc-NSIP vs fibrotic NSIP.

\section{HRCT}

All HRCT was performed with 1.0 - or $1.5-\mathrm{mm}$ thick sections at supine and full inspiration at $10-\mathrm{mm}$ intervals. A specialized chest radiologist and a pneumologist analyzed the images at three pre-established levels (trachea, carina, and pulmonary veins) for the presence of any signs of ILD: ground glass, consolidation, reticular, honeycombing, and bronchiectasia.

\section{Histological analysis}

Open lung biopsy was performed by formal thoracotomy avoiding honeycombing areas. Two pathologists specialized in lung diseases, blinded to clinical aspects of the patients, classified the lung specimens according to the new consensus on classification of ILD (25). Final diagnoses were reached by consensus of the pathologists. Regarding NSIP, the most predominant pulmonary histological pattern was also defined as cellular or fibrosing. As control, normal lung tissue was obtained from 10 individuals ( 3 males and 7 females), with a median age of 47 years (range, 31 to 60 years) who died suddenly of nonpulmonary causes.

\section{Pulmonary function tests}

Spirometric analyzes included the assessment of FEV1, FVC, and TLC. DLCO-Hb (26) was evaluated using a singlebreath technique. Results are reported as a percentage of predicted values based on gender, age, and height.

\section{Immunostaining}

A standard peroxidase technique was used, with Harris's hematoxylin as the counterstain. All of the antibodies used were biotinylated rabbit polyclonal antibodies. Neuronal NOS (nNOS), eNOS, iNOS, PAl-1, $\alpha-$ smooth muscle actin ( $\alpha-S M A), I L-4, I L-13$, and bFGF polyclonal antibodies (Santa Cruz Biotechnology, Inc., USA) were incubated with tissue sections at a 1:100 dilution. A Max Polymer Novolink amplification kit (Leica, Newcastle Inc., UK) was used for signal amplification and $3,3^{\prime}$-diaminobenzidine tetrachloride $(0.25 \mathrm{mg}$ dissolved in $1 \mathrm{~mL} 0.02 \%$ hydrogen peroxide) was used as a precipitating substrate for signal detection. The specificity of primary antibodies was confirmed by appropriate reagent controls (omitting the primary antibody or substituting 
nonimmune serum for the primary antibody in the staining protocol), which revealed no staining.

\section{Histomorphometry}

Immunohistochemical staining of NOS isoforms, PAI1, $\alpha$-SMA, IL-4, IL-13, and bFGF-positive cells in alveolar septa, as well as endothelial, myofibroblast, and smooth muscle cells in terminal bronchiolar arteries, were quantified by stereology at $400 \times$ magnification with an eyepiece systematic point-sampling grid with 100 points and 50 lines used to count the fraction of lines overlying the positively stained structures (27).

We averaged the observations from 10 microscopic fields to obtain the final results, which are reported as a percentage of the stained structures. To control for variation in scoring between our two histologists (ACAJ and ERP), $20 \%$ of the stained slides were independently scored by both observers. The coefficient of variance between cell counts for the two observers was $<5 \%$.

\section{Statistical analysis}

Data are reported as means \pm SD with $95 \%$ confidence intervals. Statistical analysis was performed by ANOVA, followed by appropriate post hoc tests, including Bonferroni's for multiple comparisons by one-way ANOVA and the Student $t$-test for two variables between groups. The general linear model was used to test the relationship between one continuous variable and several others, and the residuals were examined to ensure that they were approximately normally distributed. Survival analyses were initially done using Kaplan-Meier curves, and final multivariate analyses were done using the Cox proportional hazard model. All analyses were done with SPSS 18.0 (SPSS Inc., USA). A P value $<0.05$ was considered to be significant.

\section{Results}

\section{Clinical features}

The clinical features of the 23 patients included in this study are shown in Table 1. Age, gender, disease duration, anti-topoisomerase I positivity, MRSS, gastro-esophageal reflux symptoms, esophageal dysmotility, and dyspnea were comparable in both groups. All 23 patients had NSIP in histological examination, and the prevalence of cellular and fibrosing patterns was similar in both groups. Diffuse skin involvement was significantly associated with cellular SSc-NSIP compared to fibrotic NSIP (72 vs 33\%, P=0.01). All patients studied showed a restrictive lung function pattern characterized by a decrease in TLC (mean values were $81 \%$ of predicted in cellular SSc-NSIP and $79 \%$ of predicted in fibrotic NSIP) and an increased FEV1/FVC ratio/100 (mean values of $106 \%$ of predicted in cellular SSc-NSIP vs $108 \%$ of predicted in fibrotic NSIP). The mean predicted values of DLCO were significantly decreased in fibrotic NSIP $(55 \%)$ compared to cellular NSIP $(77 \%)$ patients (Table 1). No

Table 1. Demographic, clinical and laboratory features of systemic sclerosis patients of the cellular and fibrotic patterns.

\begin{tabular}{lcc}
\hline & Cellular pattern $(\mathrm{n}=11)$ & Fibrotic pattern $(\mathrm{n}=12)$ \\
\hline Age (years) & $42 \pm 8$ & $47 \pm 6$ \\
Gender (female) & 11 & 12 \\
Disease duration (years) & $9.83 \pm 7.79$ & $11.10 \pm 9.10$ \\
Type (limited/diffuse) & $3(27) / 8(72)$ & $8(66) / 4(33)^{*}$ \\
Scl70 & $7(63)$ & $9(75)$ \\
MRSS & $11.75 \pm 8.51$ & $20.94 \pm 14.13$ \\
GER symptoms (years) & $3.56 \pm 2.43$ & $4.06 \pm 3.97$ \\
Esophageal dysmotility and/or esophagitis & $9(81)$ & $10(83)$ \\
Dyspnea symptoms (years) & $3.50 \pm 3.37$ & $4.70 \pm 2.86$ \\
Cyclophosphamide & $0(0)$ & $2(16)$ \\
Spirometry & & $65 \pm 16.1$ \\
FEV1 (\% predicted) & $74 \pm 13.5$ & $59 \pm 14.8$ \\
FVC (\% predicted) & $70 \pm 13.9$ & $108 \pm 6.1$ \\
FEV1/FVC & $106 \pm 11.3$ & $79 \pm 13.8$ \\
TLC (\% predicted) & $81 \pm 11.1$ & $124 \pm 48.9$ \\
RV (\% predicted) & $110 \pm 14.3$ & $55 \pm 19.1^{*}$ \\
DLCO (\% predicted) & $77 \pm 14.4$ & $70 \pm 37.9$ \\
DLCO/VA (\% predicted) & $92 \pm 25.7$ & \\
\hline
\end{tabular}

Data are reported as means \pm SD or number (\%). Scl70: anti-topoisomerase I antibody; MRSS: modified Rodnan skin score; GER: gastro-esophageal reflux; FEV1: forced expiratory volume in $1 \mathrm{~s}$; FVC: forced vital capacity; TLC: total lung capacity; RV: residual volume; DLCO: diffusing capacity of the lung for carbon monoxide; VA: alveolar volume. ${ }^{*} \mathrm{P}<0.05$, compared to cellular pattern (Student $t$-test). 


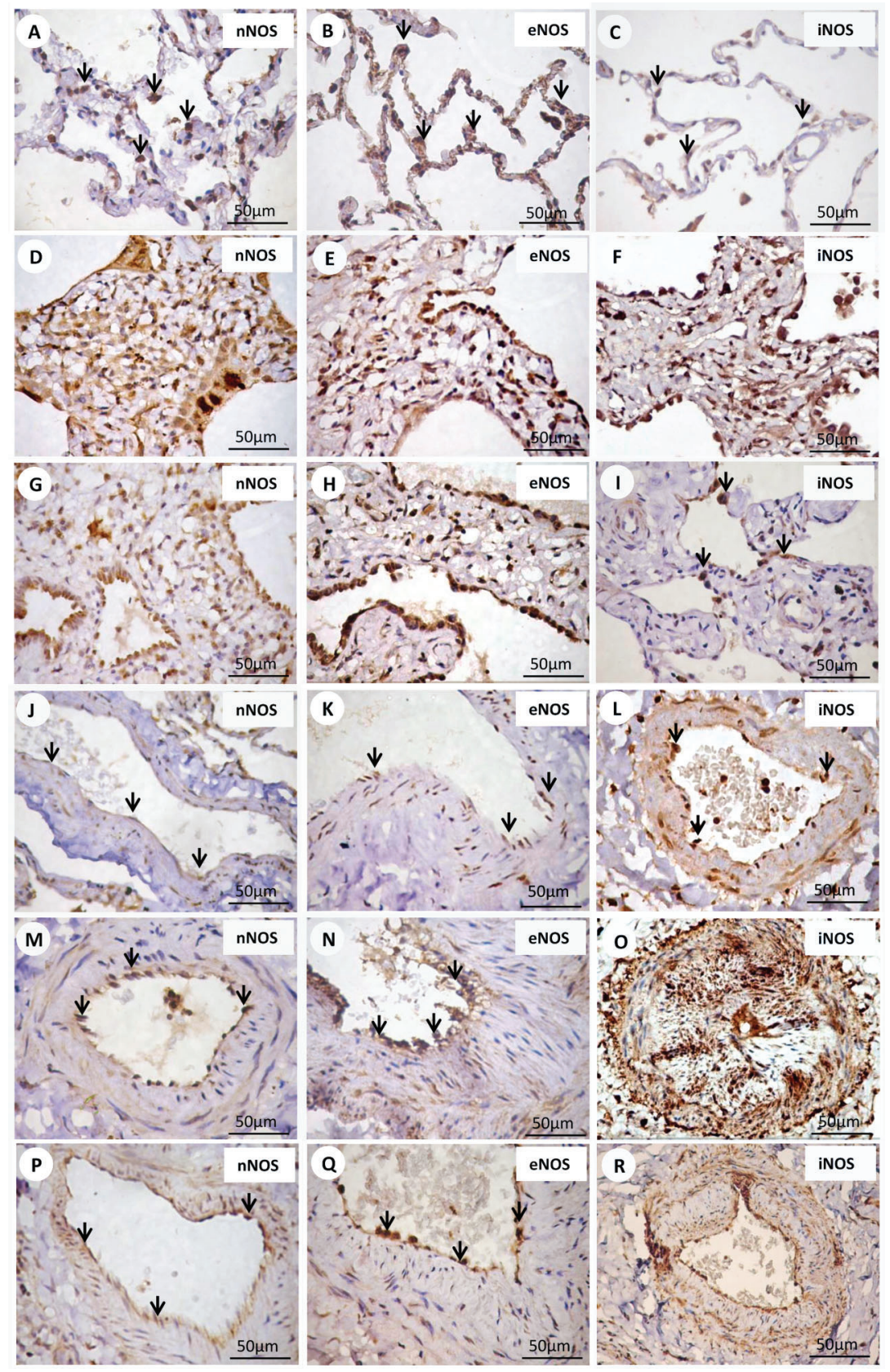

Figure 1. Immunohistochemical staining with nNOS, eNOS and iNOS. Cell expressions of nNOS, eNOS and iNOS in septal interstitium and intrapulmonary vessels from normal and systemic sclerosis (SSc) lung tissue are shown. There is a diffuse and increased expression of nNOS $(D)$, eNOS $(E)$ and iNOS $(F)$ in the septal interstitium of patients with cellular nonspecific interstitial pneumonia (NSIP) compared with nNOS (arrows) $(A)$, eNOS (arrows) $(B)$ and iNOS (arrows) $(C)$ of the control group and more than $\mathrm{nNOS}(G)$, eNOS $(H)$, and iNOS $(I)$ of fibrotic NSIP. An increased expression of iNOS in vessels of cellular NSIP $(O)$ and fibrotic NSIP $(R)$ is observed when compared with control $(L)$. Similar expression of nNOS and eNOS is observed comparing nNOS (arrows) (M) and eNOS (arrows) $(N)$ in vessels of cellular NSIP and nNOS (arrows) $(J)$ and eNOS (arrows) $(K)$ of control and nNOS (arrows) $(P)$ and eNOS (arrows) $(Q)$ of fibrotic NSIP. 

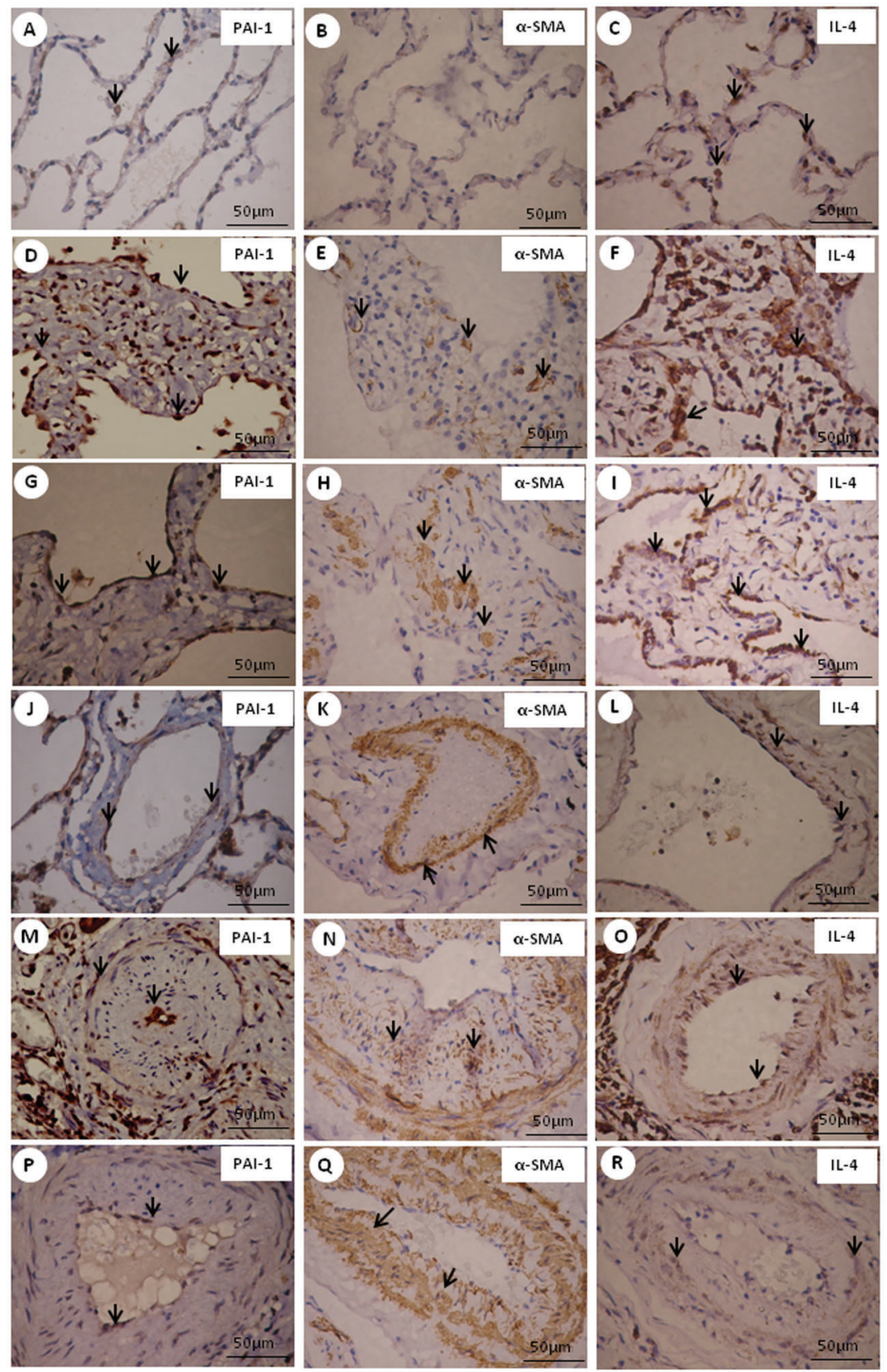

Figure 2. Immunohistochemical staining with PAI-1, $\alpha$-SMA and IL-4. Cell expressions of PAI-1, $\alpha-S M A$ and IL-4 in septal interstitium and intrapulmonary vessels from normal and systemic sclerosis (SSc) lung tissue are shown. There is a diffuse and increased expression of PAI-1 (arrows) $(D), \alpha$-SMA (arrows) $(E)$ and IL-4 (arrows) $(F)$ in the septal interstitium of cellular nonspecific interstitial pneumonia (NSIP) contrasting with a minimal or without expression of PAl-1 (arrows) $(A), \alpha-S M A(B)$ and IL-4 (arrows) (C) of the control group. Comparing the expression of PAI-1, $\alpha$-SMA, and IL-4 between the cellular $(D, E, F)$ and fibrotic $(G, H, I)$ NSIP pattern, a higher expression of $\alpha$-SMA in fibrotic than in cellular NSIP was observed. Increased expression of PAI-1, $\alpha$-SMA and IL-4 is observed in cellular (arrows) $(M, N, O)$ and fibrotic (arrows) $(P, Q, R)$ NSIP groups when compared with the control (arrows) $(J, K, L)$ group. 
difference was found for DLCO/alveolar volume in cellular SSc-NSIP compared to fibrotic NSIP (92 vs $70 \%$; $P=0.26$; Table 1).

\section{Morphological features}

Normal and NSIP histological patterns of alveolar septa and vessels are shown in Figures 1, 2, and 3, with immunohistochemical staining by nNOS (Figure 1, left panels), eNOS (Figure 1, middle panels), and iNOS (Figure 1, right panels); PAl-1 (Figure 2, left panels), $\alpha$-SMA (Figure 2, middle panels), and IL-4 (Figure 2, right panels); IL-13 (Figure 3, left panels), and bFGF (Figure 3, right panels). Different immunostaining intensities were exhibited by epithelial, endothelial, myofibroblast, and smooth muscle cells from alveolar septa and vessels in cellular SSc-NSIP histological patterns when compared to normal and fibrotic SSc-NSIP. Table 2 summarizes the morphometric results. A significant percentage of septal and vessel cells immunostained for iNOS in a cellular SSc-NSIP histological pattern $(P=0.001$ and $P=0.02$, respectively). In addition, we found that the level of staining for iNOS related significantly to several factors having to do with the immune response and fibrinolysis regulators. A general linear model analysis demonstrated that staining for septal iNOS related significantly to the staining of septal cells for IL-4 $(P=0.03)$ and to septal IL-13 $(P=0.03)$. All these relationships were significant after allowing for the contribution of the others, and for this analysis we used a multivariable model. In addition, using univariate analyses, staining for vascular iNOS related significantly to staining of vascular eNOS $(P=0.009)$, vascular PAI-1 $(P=0.003)$, and vascular IL-4 $(P=0.02)$. Also, using univariate analysis, septal and vascular iNOS were negatively related, respectively, to bFGF $(P=0.02)$ and $\alpha$-SMA $(P=0.001)$. In other words, higher levels of septal and vascular cells staining for iNOS were associated with a smaller percentage of septal and vascular cells expressing bFGF and myofibroblast proliferation, respectively. Other NOS isoforms did not relate to IL-4, IL13, PAI, and bFGF. Figure 4 uses two plots to demonstrate the relationships between staining for septal and vascular iNOS and SSc-NSIP histological patterns. The two box plots demonstrate that the relationship between iNOS and SSc-NSIP histological patterns was very strong. The scatter plots in Figure 5 show that there was a strong relationship between staining of septal iNOS and IL-4, IL-13, and bFGF, as well as between vascular iNOS and IL-4, eNOS, PAI-1, and $\alpha$-SMA. No significant association was found between staining of septal iNOS and eNOS, PAI-1, and $\alpha$-SMA; equally, no significant association between staining of vascular iNOS and IL-13 and bFGF was found.

\section{Survival analysis}

Preliminary examination of Kaplan-Meier survival curves demonstrated that, in this study, patients with

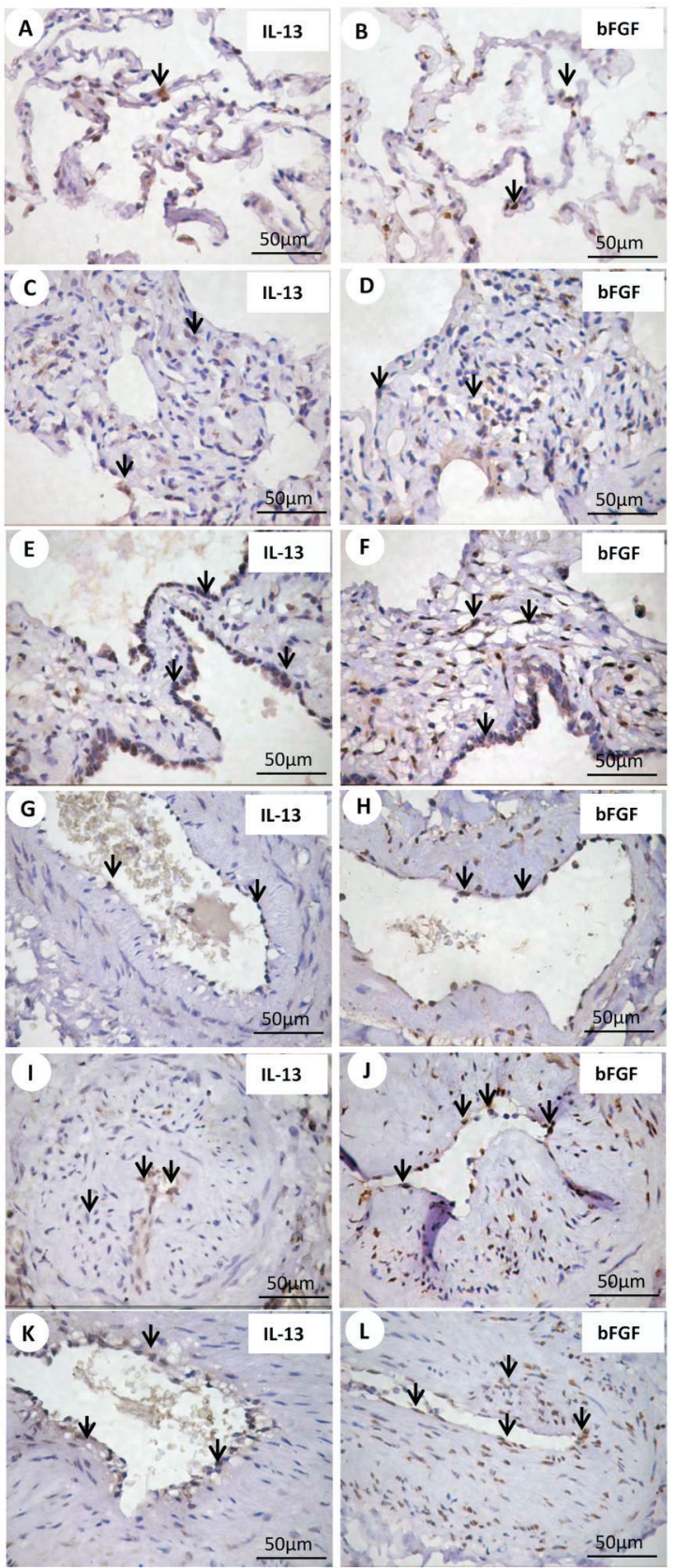

Figure 3. Immunohistochemical staining with IL-13 and bFGF. Cell expressions of IL-13 and bFGF in septal interstitium and intrapulmonary vessels from normal and systemic sclerosis (SSc) lung tissue are shown. There is an increased expression of IL-13 (arrows) $(E)$ and bFGF (arrows) $(F)$ in the septal interstitium from patients with fibrotic nonspecific interstitial pneumonia (NSIP) compared with IL-13 (arrows) $(A)$ and bFGF (arrows) (B) of the control group, and IL-13 (arrows) (C) and bFGF (arrows) (D) of the cellular pattern. Similar expression is observed comparing the IL-13 and bFGF of control (arrows) $(G, H)$, cellular (arrows) $(I, J)$, and fibrotic (arrows) $(K, L)$ groups. 
Table 2. Summary of morphometric results.

\begin{tabular}{lcccc}
\hline Variables & Normal lung tissue & Cellular SSc & Fibrotic SSc & SSc \\
\hline Septal & & & & $20.49 \pm 14.36$ \\
nNOS & $18.64 \pm 0.90$ & $25.70 \pm 18.02$ & $15.28 \pm 7.00$ & $18.32 \pm 16.24$ \\
eNOS & $10.94 \pm 2.03$ & $23.89 \pm 21.33$ & $12.75 \pm 5.54$ & $17.26 \pm 6.57$ \\
iNOS & $5.38 \pm 0.61$ & $21.07 \pm 6.42$ & $12.68 \pm 2.76$ & $13.25 \pm 5.07$ \\
PAl-1 & $8.85 \pm 1.59$ & $13.56 \pm 4.83$ & $12.84 \pm 5.61$ & $6.36 \pm 2.77$ \\
$\alpha$-SMA & $0.00 \pm 0.00$ & $5.42 \pm 2.76$ & $7.49 \pm 2.47$ & $12.79 \pm 4.57$ \\
IL-4 & $0.94 \pm 95$ & $15.50 \pm 2.81$ & $9.26 \pm 4.00$ & $3.77 \pm 2.94$ \\
IL-13 & $0.83 \pm 0.60$ & $5.46 \pm 2.45$ & $1.53 \pm 1.86$ & $3.75 \pm 2.40$ \\
bFGF & $0.67 \pm 0.23$ & $2.60 \pm 1.44$ & $5.01 \pm 2.66$ & $7.37 \pm 4.20$ \\
Vascular & & & & $10.18 \pm 7.41$ \\
nNOS & $20.00 \pm 6.34$ & $9.75 \pm 4.08$ & $4.51 \pm 2.04$ & $17.46 \pm 7.71$ \\
eNOS & $7.68 \pm 2.97$ & $13.82 \pm 8.73$ & $6.54 \pm 3.16$ & $14.42 \pm 14.56$ \\
iNOS & $3.92 \pm 0.77$ & $20.83 \pm 8.19$ & $13.40 \pm 4.82$ & $63.41 \pm 12.91$ \\
PAI-1 & $4.35 \pm 0.86$ & $18.03 \pm 18.72$ & $72.70 \pm 7.72$ & $15.52 \pm 9.17$ \\
$\alpha-S M A$ & $9.57 \pm 2.90$ & $54.11 \pm 10.03$ & $8.22 \pm 4.75$ & $2.19 \pm 4.28$ \\
IL-4 & $1.86 \pm 0.75$ & $20.21 \pm 8.25$ & $2.51 \pm 4.92$ & $4.38 \pm 2.48$ \\
IL-13 & $0.54 \pm 0.65$ & $1.92 \pm 3.88$ & $5.81 \pm 2.37$ & \\
bFGF & $0.99 \pm 0.68$ & $3.08 \pm 1.81$ & & \\
\hline
\end{tabular}

Data are reported as "\% of points", which is the number of points overlying the phenomena of interest divided by the total number of points overlying septal and vascular areas. In morphometry, this is called a point fraction and is often symbolized as Pp. Pp has been shown to approximate the volume fraction or Vv. SSc: systemic sclerosis.

fibrotic NSIP, septal iNOS $<17.26 \%$, septal IL-13 $<5.46 \%$, vascular nNOS $<7.37 \%$, vascular $\alpha$-SMA $>63.4 \%$, and vascular IL-4 $<12.79 \%$ had approximately the same hazard for survival with a median survival time equal to 49.5 months for all these variables. Thus, we coded overall NSIP histological patterns as a single dummy variable with a value of zero for cellular and a value of one for fibrotic. The results of the Cox model analysis are reported in Table 3. After controlling for the SSc-NSIP histological pattern, only three variables were significantly associated with survival time: septal iNOS $(P=0.04)$, septal IL-13 $(P=0.03)$, and septal bFGF $(P=0.02)$. Once these three variables was accounted
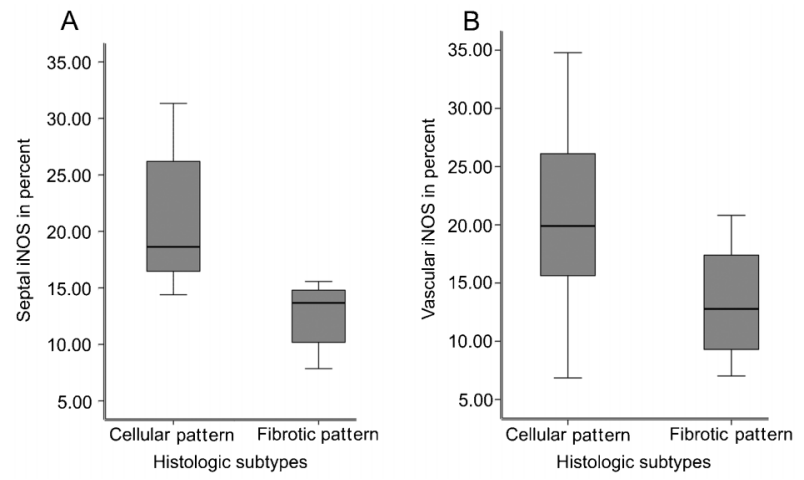

Figure 4. Box plot shows septal $(A)$ and vascular $(B)$ iNOS expression between cellular and fibrotic histological patterns. for, none of the others related to survival. Multivariate analyses showed low risk of death for low septal iNOS, septal IL-13, and septal bFGF expression.

\section{Discussion}

We demonstrated higher expressions of iNOS in alveolar and vascular structures in patients with SSc when compared with the normal lung tissue group. Alveolar structures and vessels had a high expression of iNOS in epithelial, endothelial, myofibroblasts, and smooth muscle cells. When total iNOS and NSIP histological patterns were compared, a clear switch was shown in the expression of the iNOS isoform in septal and vascular lesions of patients with SSc. Although the expression data were similar between iNOS in septal and vascular cells, the following features were constant: iNOS was upregulated in epithelial, endothelial, myofibroblasts, and smooth muscle cells from septa and vessels; and the expression of iNOS was more strongly associated with higher pulmonary fibrosis in SSc. One interpretation of the relative pattern of expression and correlation of iNOS is that, as pulmonary lesions in patients with severe pulmonary fibrosis become more extensive, more extrinsic or intrinsic stimuli cause endothelial upregulation of iNOS production and more endothelial injury than in patients with minimal pulmonary fibrosis. This in turn leads to production of sufficient amounts of NO to cause NO-mediated free radical damage to proteins within endothelial and smooth muscle cells in patients with 

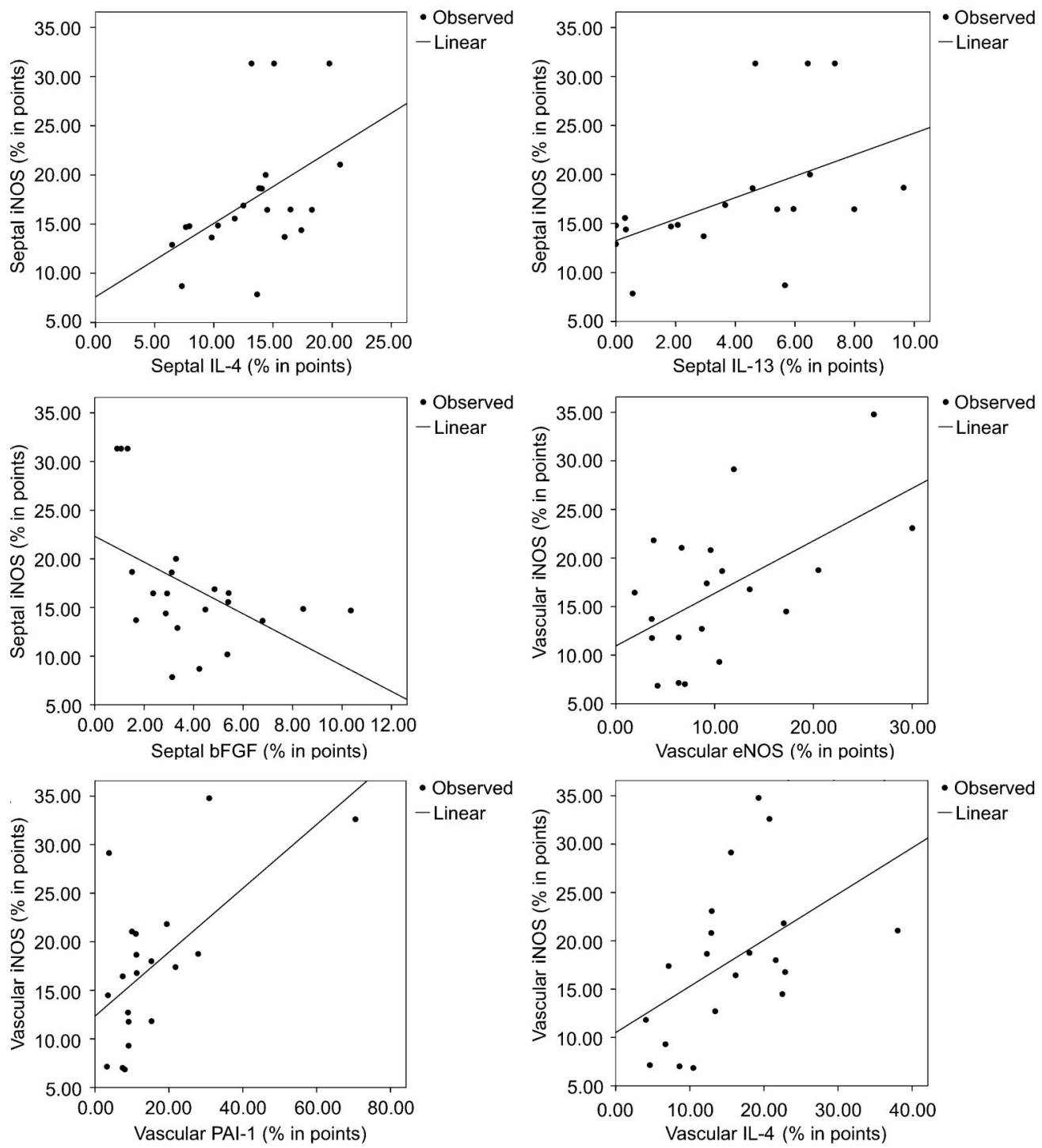

- Observed
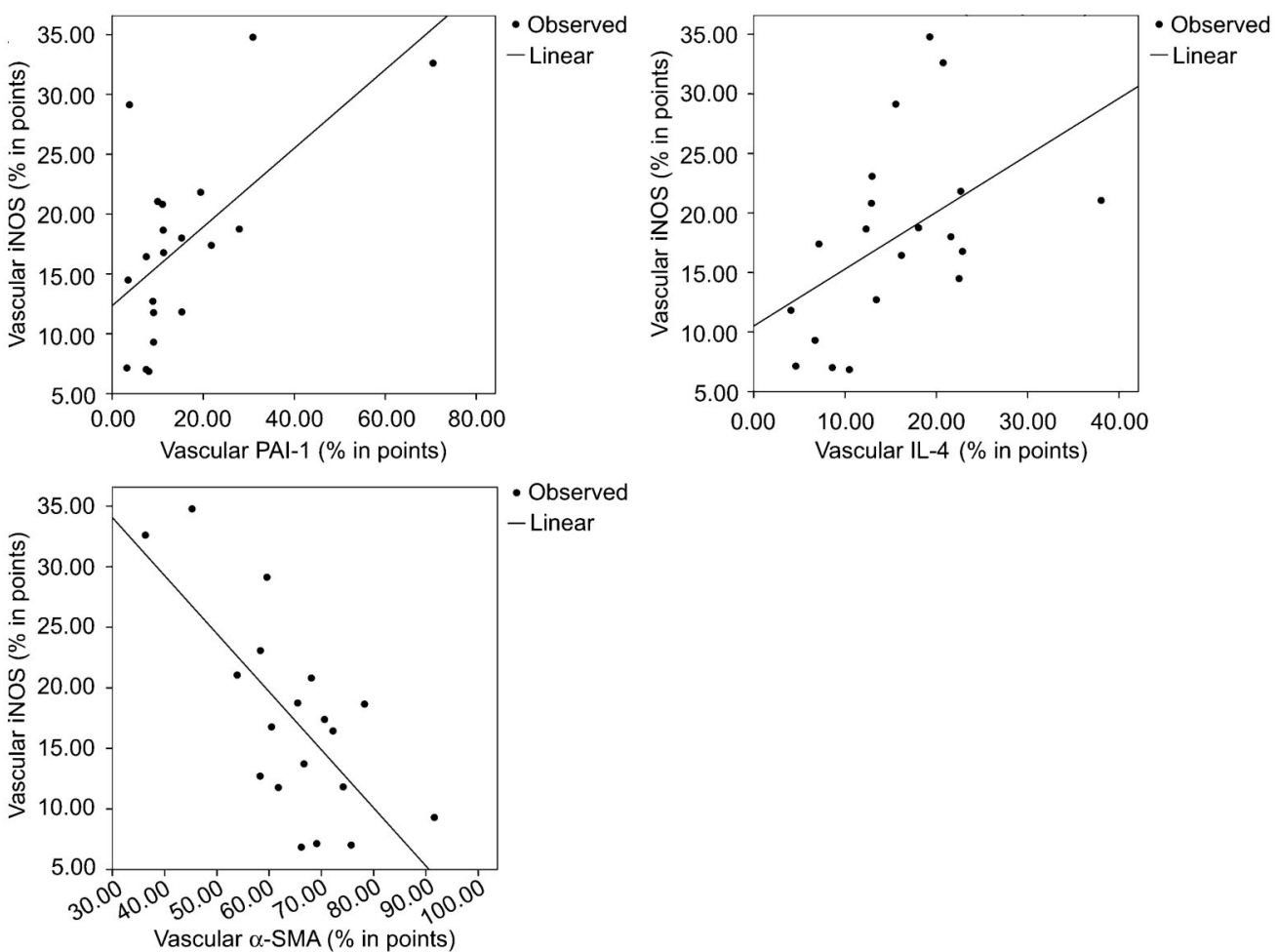

Figure 5. Scatter plots show the relationship between staining of septal and vascular cells for iNOS and IL-4, IL-13, bFGF, eNOS, PAI-1, and $\alpha$-SMA. 
Table 3. Cox proportional hazards regression to ascertain the individual contribution of histological subtypes and morphological factors associated with survival and to compare adjusted survival between the groups [ -2 log likelihood $=35.15$; chi square $=21.36 ; P<0.002]$.

\begin{tabular}{lrrrrrrr}
\hline & $\beta$ & SE & Wald test & $P$ & Exp $(\beta)$ & \multicolumn{2}{c}{$95 \% \mathrm{Cl}$ for Exp ( $\beta$ ) } \\
\cline { 5 - 8 } & & & & & & Lower & Upper \\
\hline Histological subtypes & 3.64 & 1.93 & 3.55 & 0.06 & 38.14 & 0.86 & 1683.89 \\
nNOS & 0.03 & 0.02 & 1.11 & 0.29 & 1.02 & 0.97 & 1.08 \\
iNOS & -0.24 & 0.12 & 3.91 & 0.04 & 0.78 & 0.61 & 0.99 \\
IL-4 & -0.18 & 0.20 & 0.85 & 0.35 & 0.82 & 0.55 & 1.23 \\
IL-13 & 0.77 & 0.35 & 4.64 & 0.03 & 2.16 & 1.07 & 4.35 \\
bFGF & 1.05 & 0.48 & 4.76 & 0.02 & 2.85 & 1.11 & 7.34 \\
\hline
\end{tabular}

extensive pulmonary damage, which can be recognized as accumulation of these proteins and an increase in the expression of PAI-1. An imbalance in the equilibrium of iNOS and other isoform (nNOS and eNOS) synthesis and the resulting increased production of NO have been reported to be associated with serious cell damage (28). In the present study, the grades at which greatest endothelial damage occurred were the same as those in which morphological studies from this laboratory have shown evidence of endothelial injury and death (18). In other situations, such as vascular changes associated with endotoxic shock (29), and ischemia-reperfusion injuries (30), endothelial iNOS expression has been associated with endothelial damage mediated by free radicals. All of our data indicate a similar process in patients with extensive pulmonary fibrosis, and this situation contributes to maintenance of the disease. The conclusion that free radical-mediated oxidative injury is involved in the progression of SSc is supported by the increase in iNOS and reduced circulating levels of antioxidants (selenium and ascorbic acid) in these patients. This situation is indicative of the formation of peroxynitrite, nitration of cellular proteins, and cell damage. The switch from upregulation of iNOS in endothelial and smooth muscle cells is not unique to SSc, having been described in other collagen vascular diseases (31). Increased expression of iNOS by endothelial cells has also been described in patients with systemic lupus erythematosus (32), but in that study there was no reduction in iNOS expression.

There is a growing body of evidence that cytokines, such as IL- $1 \alpha$, TNF- $\alpha$, TGF- $\alpha$, IFN- $\alpha$, and bFGF, and other local effectors, such as heparin, lipopolysaccharide, and ischemia, might be involved in the regulation of iNOS isoform expression in the endothelium (16,33-35). Our results support the hypothesis that NO production

\section{References}

1. Steen VD. Clinical manifestations of systemic sclerosis. Semin Cutan Med Surg 1998; 17: 48-54, doi: 10.1016/ S1085-5629(98)80062-X. following induction of vascular iNOS contributes to free radical damage previously implicated in the pathogenesis of SSc. One implication of these findings is that general stimulation of NO production in patients with SSc through vasodilatation improves tissue blood flow, and thus cell viability, and could be counterproductive unless therapy is first directed toward selective inhibition of these isoforms. Such selective inhibition can diminish endothelial damage that occurs in progressive pulmonary fibrosis in SSc.

Our study presented clinical and functional impacts. We found an important correlation between pulmonary function tests and high compromise by pulmonary fibrosis in these patients. In order to establish the relevance of these findings to the evolution of the patients, NOS and cytokines were evaluated in the function of survival controlled for histological patterns. Clearly, multivariate analyses showed a low risk of death for low expressions of septal iNOS, septal IL-13, and bFGF.

We conclude that iNOS, IL-13, and bFGF expression in lung parenchyma offers us the potential to control oxidative injury involved in fibrotic progression of SSc, suggesting that strategies aimed at preventing high iNOS synthesis, or local responses to high IL-13 and bFGF cytokines may have a greater impact on SSc. To finalize this conclusion will require greater study in a randomized and prospective trial.

\section{Acknowledgments}

We are grateful to the biologist Sandra de Morais Fernezlian from the Immunohistochemistry Laboratory of the Department of Pathology (Faculdade de Medicina da Universidade de São Paulo) for technical assistance. Research supported by CNPq and FAPESP (Project \#2008/53022-3). 
3. Jacobsen S, Ullman S, Shen GQ, Wiik A, Halberg P. Influence of clinical features, serum antinuclear antibodies, and lung function on survival of patients with systemic sclerosis. J Rheumatol 2001; 28: 2454-2459.

4. Steen VD, Medsger TA Jr. Severe organ involvement in systemic sclerosis with diffuse scleroderma. Arthritis Rheum 2000; 43: 2437-2444, doi: 10.1002/1529-0131(200011) 43:11<2437::AID-ANR10>3.0.CO;2-U.

5. Altman RD, Medsger TA Jr, Bloch DA, Michel BA. Predictors of survival in systemic sclerosis (scleroderma). Arthritis Rheum 1991; 34: 403-413, doi: 10.1002/ art. 1780340405.

6. Hubbard R, Venn A. The impact of coexisting connective tissue disease on survival in patients with fibrosing alveolitis. Rheumatology 2002; 41: 676-679, doi: 10.1093/ rheumatology/41.6.676.

7. Steen VD, Conte C, Owens GR, Medsger TA Jr. Severe restrictive lung disease in systemic sclerosis. Arthritis Rheum 1994; 37: 1283-1289, doi: 10.1002/art.1780370903.

8. Felicio $\mathrm{CH}$, Parra ER, Capelozzi VL. Idiopathic and collagen vascular disease nonspecific interstitial pneumonia: clinical significance of remodeling process. Lung 2007; 185: 39-46, doi: 10.1007/s00408-006-0104-2.

9. Rozin GF, Gomes MM, Parra ER, Kairalla RA, de Carvalho $\mathrm{CR}$, Capelozzi VL. Collagen and elastic system in the remodelling process of major types of idiopathic interstitial pneumonias (IIP). Histopathology 2005; 46: 413-421, doi: 10.1111/j.1365-2559.2005.02103.x.

10. Baptista AL, Parra ER, Barbas Filho JV, Kairalla RA, de Carvalho CR, Capelozzi VL. Structural features of epithelial remodeling in usual interstitial pneumonia histologic pattern. Lung 2006; 184: 239-244, doi: 10.1007/s00408-005-2585-9.

11. Parra ER, Silverio da Costa LR, Ab'Saber A, Ribeiro de Carvalho CR, Kairalla RA, Fernezlian SM, et al. Nonhomogeneous density of CD34 and VCAM-1 alveolar capillaries in major types of idiopathic interstitial pneumonia. Lung 2005; 183: 363-373, doi: 10.1007/s00408-005-2548-1.

12. Parra ER, Kairalla RA, de Carvalho CR, Capelozzi VL. Abnormal deposition of collagen/elastic vascular fibres and prognostic significance in idiopathic interstitial pneumonias. Thorax 2007; 62: 428-437, doi: 10.1136/thx.2006.062687.

13. Hill MB, Phipps JL, Cartwright RJ, Milford WA, Greaves M, Hughes $P$. Antibodies to membranes of endothelial cells and fibroblasts in scleroderma. Clin Exp Immunol 1996; 106: 491-497, doi: 10.1046/j.1365-2249.1996.d01-867.x.

14. Bandinelli F, Bartoli F, Perfetto E, Del Rosso A, MoggiPignone A, Guiducci $S$, et al. The fibrinolytic system components are increased in systemic sclerosis and modulated by Alprostadil (alpha1 ciclodestryn). Clin Exp Rheumatol 2005; 23: 671-677.

15. Liu JS, Zhao ML, Brosnan CF, Lee SC. Expression of inducible nitric oxide synthase and nitrotyrosine in multiple sclerosis lesions. Am J Pathol 2001; 158: 2057-2066, doi: 10.1016/S0002-9440(10)64677-9.

16. MacNaul KL, Hutchinson NI. Differential expression of iNOS and cNOS mRNA in human vascular smooth muscle cells and endothelial cells under normal and inflammatory conditions. Biochem Biophys Res Commun 1993; 196: 1330-1334, doi: 10.1006/bbrc.1993.2398.

17. Gutierrez HH, Pitt BR, Schwarz M, Watkins SC, Lowenstein C, Caniggia I, et al. Pulmonary alveolar epithelial inducible
NO synthase gene expression: regulation by inflammatory mediators. Am J Physiol 1995; 268: L501-L508.

18. Parra ER, Aguiar AC Jr, Teodoro WR, de Souza R, Yoshinari NH, Capelozzi VL. Collagen V and vascular injury promote lung architectural changes in systemic sclerosis. Clin Respir J 2009; 3: 135-142, doi: 10.1111/j.1752-699X. 2008.00118.x.

19. Allanore $Y$, Dieude $P$, Boileau C. Genetic background of systemic sclerosis: autoimmune genes take centre stage. Rheumatology 2010; 49: 203-210, doi: 10.1093/rheumatology/ kep368.

20. Muller-Ladner U, Distler O, Ibba-Manneschi L, Neumann E, Gay S. Mechanisms of vascular damage in systemic sclerosis. Autoimmunity 2009; 42: 587-595, doi: 10.1080/ 08916930903002487

21. de Souza RB, Borges CT, Capelozzi VL, Parra ER, Jatene FB, Kavakama J, et al. Centrilobular fibrosis: an underrecognized pattern in systemic sclerosis. Respiration 2009; 77: 389-397, doi: 10.1159/000156958.

22. Subcommittee for scleroderma criteria of the American Rheumatism Association Diagnostic and Therapeutic Criteria Committee. Preliminary criteria for the classification of systemic sclerosis (scleroderma). Arthritis Rheum 1980; 23: 581-590, doi: $10.1002 /$ art. 1780230510.

23. LeRoy EC, Black C, Fleischmajer R, Jablonska S, Krieg T, Medsger TA Jr, et al. Scleroderma (systemic sclerosis): classification, subsets and pathogenesis. I Rheumatol 1988; 15: 202-205.

24. Furst DE, Clements PJ, Steen VD, Medsger TA Jr, Masi AT, D'Angelo WA, et al. The modified Rodnan skin score is an accurate reflection of skin biopsy thickness in systemic sclerosis. J Rheumatol 1998; 25: 84-88.

25. American Thoracic Society, European Respiratory Society. American Thoracic Society/European Respiratory Society International Multidisciplinary Consensus Classification of the Idiopathic Interstitial Pneumonias. This joint statement of the American Thoracic Society (ATS), and the European Respiratory Society (ERS) was adopted by the ATS board of directors, June 2001 and by the ERS Executive Committee, June 2001. Am J Respir Crit Care Med 2002; 165: 277-304, doi: 10.1164/ajrccm.165.2.ats01.

26. Cotes JE, Dabbs JM, Elwood PC, Hall AM, McDonald A, Saunders MJ. Iron-deficiency anaemia: its effect on transfer factor for the lung (diffusiong capacity) and ventilation and cardiac frequency during sub-maximal exercise. Clin Sci 1972; 42: 325-335.

27. Hsia CC, Hyde DM, Ochs M, Weibel ER. An official research policy statement of the American Thoracic Society/European Respiratory Society: standards for quantitative assessment of lung structure. Am J Respir Crit Care Med 2010; 181: 394418, doi: $10.1164 / \mathrm{rccm} .200809-1522 \mathrm{ST}$.

28. Peng TI, Jou MJ. Oxidative stress caused by mitochondrial calcium overload. Ann N Y Acad Sci 2010; 1201: 183-188, doi: 10.1111/j.1749-6632.2010.05634.x.

29. Miranda LE, Capellini VK, Reis GS, Celotto AC, Carlotti CG Jr, Evora PR. Effects of partial liver ischemia followed by global liver reperfusion on the remote tissue expression of nitric oxide synthase: lungs and kidneys. Transplant Proc 2010; 42: 1557-1562, doi: 10.1016/j.transproceed.2010.02.097.

30. Lin HI, Wang D, Leu FJ, Chen CF, Chen HI. Ischemia and reperfusion of liverinduces eNOS andiNOS expression: effects of 
a NO donor and NOS inhibitor. Chin J Physiol 2004; 47: 121-127.

31. Nagy G, Koncz A, Telarico T, Fernandez D, Ersek B, Buzas E, et al. Central role of nitric oxide in the pathogenesis of rheumatoid arthritis and systemic lupus erythematosus. Arthritis Res Ther 2010; 12: 210, doi: 10.1186/ar3045.

32. Belmont HM, Levartovsky D, Goel A, Amin A, Giorno R, Rediske J, et al. Increased nitric oxide production accompanied by the up-regulation of inducible nitric oxide synthase in vascular endothelium from patients with systemic lupus erythematosus. Arthritis Rheum 1997; 40: 1810-1816, doi: 10.1002/art.1780401013.

33. Hung LF, Lai JH, Lin LC, Wang SJ, Hou TY, Chang DM, et al. Retinoid acid inhibits IL-1-induced iNOS, COX-2 and chemokine production in human chondrocytes. Immunol Invest 2008; 37: 675-693, doi: 10.1080/0882013080230 7237.

34. Yague S, Alvarez Arroyo V, Castilla A, Gonzalez Pacheco FR, Llamas P, Caramelo C. Modulation of the effect of vascular endothelial growth factor on endothelial cells by heparin: critical role of nitric oxide-mediated mechanisms. J Nephrol 2005; 18: 234-242.

35. Farrell AJ, Blake DR, Palmer RM, Moncada S. Increased concentrations of nitrite in synovial fluid and serum samples suggest increased nitric oxide synthesis in rheumatic diseases. Ann Rheum Dis 1992; 51: 1219-1222, doi: 10. 1136/ard.51.11.1219. 